\title{
Examination of Polycrystalline Diamond Compact Cutter used in Drilling Tools in the Oil Industry
}

\author{
John N. Williard ${ }^{1,}$ Donna K. Colbert ${ }^{1}$ \\ 1. Baker Hughes, 9110 Grogan's Mill Road, The Woodlands, TX 77380
}

Characterization of the dimensional, surface and chemical microstructure of polycrystalline diamond compact (PDC) cutters used in the drilling industry involves the examination of the synthetic diamonds bonded to a cemented tungsten carbide substrate. This presentation will cover recent microscopy developments with special emphasis on other non-destructive analytical techniques such as scanning acoustic microscope (CSAM), SmartScope Flash, and color 3-D laser scanning microscopy (LSM). Examples and applications of materials used during PDC drill bit manufacture and forensic analysis will be examined. The results of these studies will assist in the design of new, innovative diamond tools as well as leading to continuing improvements in bit performance in drilling operations.

A great deal of research has already been undertaken to understand the properties and defect structures of natural and synthetic diamond grains. What emerges from these fundamental studies is that the properties of synthetic diamonds can vary and should be considered as part of a multi-component system requiring the successful performance of many subsystems. These subsystems include: the diamond grains themselves, the binder phase or phases used in the sintering operation to form the composite and the method of bonding the composite element into the body of the tool [1-3].

A typical PDC cutter comprises a thin layer of sintered polycrystalline diamond bonded to a tungsten carbide-cobalt substrate, see Figure 1. A well-recognized failure mechanism is delamination at the interface between diamond and cemented carbide [4]. SEM and scanning acoustic microscopy (C-SAM) were used to examine cutters after drilling, see Figures 2-3. C-SAM is used for the detection of three major types of defect such as diamond layer debonding from the substrate, crack and thermal defects of the diamond layers [5].

Dimensional measurements of the PDC are another key factor in quality drill bit manufacturing. Using the SmartScope Flash enables automated dimensional measuring to ensure the PDC meets specification, see Figures 4-5. Three different light sources enable measuring all dimensions called out in the specified drawings.

The laser scanning microscope (LSM) provides non-destructive, non-contact highaccuracy measurements with a pinhole confocal optical system. This system can be used to perform height, profile, area, 3-D image, and surface topography measurements, see Figure 6. The system has a laser resolution of $0.4 \mathrm{um}$ as part of the standard multi-plane LSM image acquisition technique. Digital stage control resolves narrow field-of-view issues with image stitching. 
Various forms of microscopy and other non-traditional approaches are yielding information through non-destructive characterizations and examination of PDC cutters. SEM/EDS techniques have been used traditionally, however; other techniques such as scanning acoustic microscope (C-SAM), SmartScope Flash, and laser scanning microscopy (LSM) are valuable tools to gain information on properties of highperformance drilling tools.

\section{References}

[1] Boland.J., et.al., Materials 2010, 3, 1390-1419.

[2] Scott D., Industrial Diamond Review 2006 No.1, p48.

[3] Mainwood, et al., J. Phys.:Condens. Matter 2009, 21, 360301.

[4] Huang J., et al.; Journal of Material Science 4 (1993), 1074-1080.

[5] Delebarre C., et al., NDT \& E International 26 (1993) 303-308.

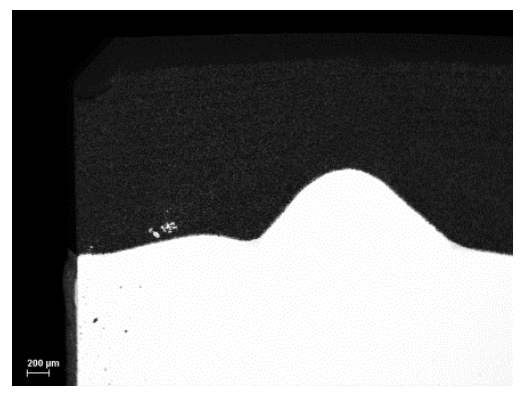

Fig 1

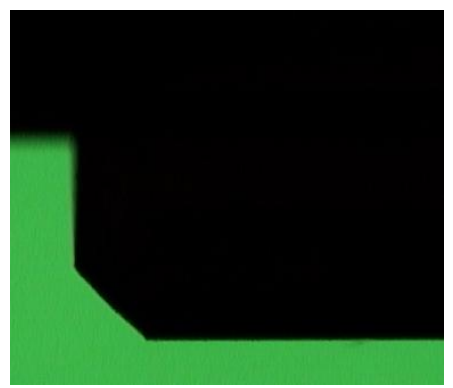

Fig 4

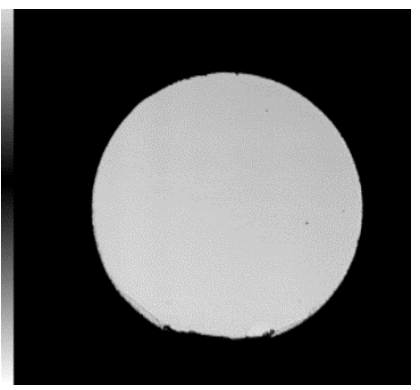

Fig 2

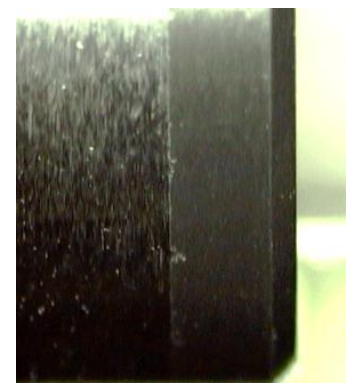

Fig 5

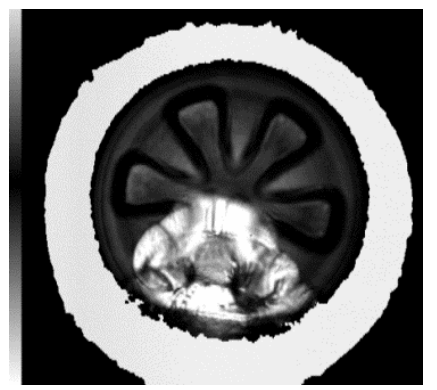

Fig 3

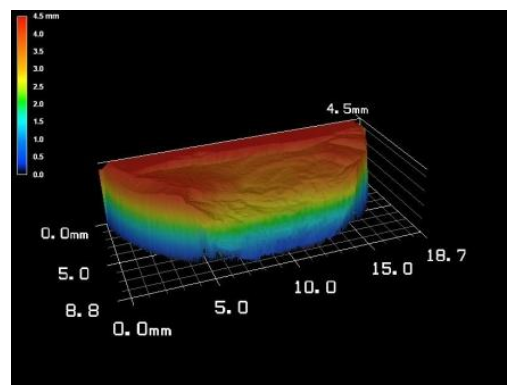

Fig 6

FIG 1. BSE image of sectioned PDC cutter showing the diamond table and substrate.

FIG. 2. C-SAM image of top of diamond table after use. Please note intact appearance of the surface

FIG 3. C-SAM image of PDC cutter interface revealing subsurface defect and disbonding. Please note performance of interface and intact bonds.

FIG 4. SmartScope Flash image of chamfer angle using back lighting.

FIG 5. SmartScope Flash image showing diamond table and carbide barrel. Ring lighting produces a 3-D image capturing heights, depths, and surface imperfections. FIG 6. 3D image produced by Laser Scanning Microscope illustrating non-contact profile and roughness on the wear scar of a PDC cutter. 